\title{
Regulation of phenylpropanoid biosynthesis by MdMYB88 and MdMYB124 contributes to pathogen and drought resistance in apple
}

Dali Geng ${ }^{1}$, Xiaoxia Shen ${ }^{1}$, Yinpeng Xie', Yusen Yang ${ }^{1}$, Ruiling Bian², Yuqi Gao ${ }^{2}$, Pengmin Li' ${ }^{1}$ Liying Sun², Hao Feng ${ }^{2}$, Fengwang $\mathrm{Ma}^{1}$ and Qingmei Guan ${ }^{1}$

\begin{abstract}
MdMYB88 and MdMYB124 have been demonstrated to be responsible for lignin accumulation in apple under drought stress. In this study, using a metabolomic approach, we identified differentially accumulated phenylpropanoid and flavonoid metabolites in MdMYB88/124 transgenic RNAi plants under control and long-term drought stress conditions in apple roots. We confirmed the regulation of phenylalanine by MdMYB88 and MdMYB124 via UPLC-MS in apple roots under both control and drought conditions. Using Electrophoretic Mobility Shift Assay (EMSA) and ChIPquantitative PCR (qPCR) analyses, we found that MdMYB88 positively regulates the MdCM2 gene, which is responsible for phenylalanine biosynthesis, through binding to its promoter region. Under long-term drought conditions, MdMYB88/124 RNAi plants consistently accumulated increased amounts of $\mathrm{H}_{2} \mathrm{O}_{2}$ and MDA, while MdMYB88 and MdMYB124 overexpression plants accumulated decreased amounts of $\mathrm{H}_{2} \mathrm{O}_{2}$ and MDA. We also examined the accumulation of metabolites in the phenylpropanoid biosynthesis pathway in the leaves of MdMYB88 and MdMYB124 transgenic apple plants after long-term drought stress. We found that metabolites responsible for plant defense, including phenylpropanoids and flavonoids, accumulated less in the RNAi plants but more in the overexpression plants under both control and drought conditions. We further demonstrated that MdMYB88/124 RNAi plants were more sensitive to Alternaria alternata f. sp. mali and Valsa mali, two pathogens that currently severely threaten apple production. In contrast, MdMYB88 and MdMYB124 overexpression plants were more tolerant to these pathogens. The cumulative results of this study provided evidence for secondary metabolite regulation by MdMYB88 and MdMYB124, further explained the molecular roles of MdMYB88 and MdMYB124 in drought resistance, and provided information concerning molecular aspects of their roles in disease resistance.
\end{abstract}

\section{Introduction}

Previous research has shown that two atypical and paralogous MYB transcription factors, namely, MdMYB88 and MdMYB124, are responsible for freezing and drought stress tolerance of apple plants ${ }^{1,2}$. In addition, MdMYB88 and MdMYB124 can also regulate the

\footnotetext{
Correspondence: Qingmei Guan (qguan@nwafu.edu.cn)

${ }^{1}$ State Key Laboratory of Crop Stress Biology for Arid Areas/Shaanxi Key Laboratory of Apple, College of Horticulture, Northwest A\&F University, Yangling, Shaanxi 712100, China

${ }^{2}$ State Key Laboratory of Crop Stress Biology for Arid Areas, College of Plant Protection, Northwest A\&F University, Yangling, Shaanxi 712100, China

These authors contributed equally: Dali Geng, Xiaoxia Shen.
}

biosynthesis of lignin under drought stress by directly targeting MdMYB46 and MdVND6, two important regulators of the biosynthesis of secondary cell wall components $^{2}$. In plants, lignin biosynthesis occurs via the phenylpropanoid biosynthesis pathway ${ }^{3}$; therefore, it is compelling to know whether MdMYB88 and MdMYB124 regulate lignin biosynthesis via the regulation of phenylpropanoid biosynthesis. Additionally, whether the biosynthesis of other secondary metabolites is regulated by MdMYB88 and MdMYB124 is important to further understand their roles in stress responses.

\section{(c) The Author(s) 2020}

(c) (i) Open Access This article is licensed under a Creative Commons Attribution 4.0 International License, which permits use, sharing, adaptation, distribution and reproduction c. in any medium or format, as long as you give appropriate credit to the original author(s) and the source, provide a link to the Creative Commons license, and indicate if changes were made. The images or other third party material in this article are included in the article's Creative Commons license, unless indicated otherwise in a credit line to the material. If material is not included in the article's Creative Commons license and your intended use is not permitted by statutory regulation or exceeds the permitted use, you will need to obtain permission directly from the copyright holder. To view a copy of this license, visit http://creativecommons.org/licenses/by/4.0/. 
The phenylpropanoid biosynthesis pathway begins with phenylalanine, which can be converted into aromatic compounds, including benzenoids, coumarins, flavonoids, hydroxycinnamates, and lignin ${ }^{4}$. These compounds often participate in plant development ${ }^{5}$ and plant-environment interactions ${ }^{6}$. Phenylalanine is synthesized from the shikimate pathway, which requires the participation of chorismate mutase $(\mathrm{CM})^{4,7}$. In Petunia hybrida cv. Mitchell, two CMs at different loci are directly responsible for the biosynthesis of phenylalanine $e^{7,8}$. In addition, the expression level of $C M s$ can substantially influence the accumulation of phenylalanine, which further affects the biosynthesis of phenylpropanoids in plants ${ }^{9-13}$.

Many phenylpropanoids and flavonoids are involved in the responses to oxidative stress ${ }^{14-16}$, drought stress ${ }^{17}$, and disease resistance ${ }^{18-21}$. In Vitis vinifera, genes related to phenylpropanoid metabolism have been found to be less induced and/or more repressed in drought-sensitive rootstocks ${ }^{22}$. Research has also shown that phenylpropanoids and flavonoids play critical roles in the response to ozone-induced oxidative stress, as relatively high contents of phenylpropanoids and flavonoids in Fraxinus excelsior L. lead to relatively low-ozone sensitivity ${ }^{23}$. In addition, phenylpropanoids play a prominent role in the response to drought-induced oxidative stress in Platanus $\times$ acerifolia. The great investment of Platanus $\times$ acerifolia in phenylpropanoid biosynthesis complements the decrease in insufficient amounts of antioxidants during drought stress $^{24}$. Phenylpropanoids and flavonoids also participate in the response to Alternaria alternata f. sp. mali. Higher contents of phenylpropanoids and flavonoids can enhance the resistance of tomatoes to A. alternata ${ }^{25}$. The defense against Valsa mali in plants also requires phenylpropanoids and flavonoids ${ }^{26}$. It has been shown that transgenic MdUGT88F1 (a key UDP-glucose:phloretin 2'-O-glucosyltransferase gene) apple plants with increased phloridzin have enhanced resistance to Valsa canker ${ }^{27}$.

Abiotic and biotic stresses, including drought and disease, have been threatening apple growth and production worldwide. Drought often results in reduced photosynthesis and solute transport, loss of turgidity, membrane lipid peroxidation, and other problems in apple ${ }^{27}$. Membrane lipid peroxidation during drought stress always leads to secondary oxidative stress ${ }^{28}$. An increase in the levels of reactive oxygen species (ROS), such as hydrogen peroxide $\left(\mathrm{H}_{2} \mathrm{O}_{2}\right)$, is the most direct indicator of oxidative stress, and malondialdehyde (MDA) is the product of membrane lipid peroxidation ${ }^{28}$. Disease caused by A. alternata f. sp. mali and Valsa mali affect apple trees by infecting the leaves and young shoots and subsequently causing defoliation, which leads to premature fruit drop $^{29-32}$. A number of studies have characterized genes important for the resistance of apple to drought and pathogens and have examined their potential use in the breeding of resistant apple $\mathrm{e}^{33-37}$. MYB88 and MYB124 and their homologous genes in apple participate in the response to salt, cold, and drought stress ${ }^{1,2,38-41}$. In the current study, we revealed that MdMYB88 and MdMYB124 regulate the accumulation of metabolites involved in the phenylpropanoid pathway by directly regulating the expression of $M d C M 2$, which contributes to pathogen defense and drought stress tolerance.

\section{Results \\ MdMYB88 and MdMYB124 regulate secondary metabolite accumulation in apple roots}

Previously, we determined that MdMYB88 and MdMYB124 regulate root lignin deposition under drought stress. Here, we carried out a secondary metabolic profiling analysis to identify additional secondary metabolites regulated by MdMYB88 and MdMYB124 under both control and drought conditions. We used an ultra-performance liquid chromatography-tandem mass spectrometry (UPLC-MS) method in conjunction with previously generated $M d M Y B 88 / 124$ RNAi plants in which both $M d M Y B 88$ and $M d M Y B 124$ were knocked down ${ }^{1}$. The results showed that a total of 678 metabolites were detected with this approach. Among these metabolites, 58 were flavone metabolites, 158 were flavonoid metabolites (including 13 catechins and catechin derivatives), 15 were anthocyanins and anthocyanin derivatives, 33 were flavonols, 29 were flavonoid glycosides, 19 were flavanones, 19 were quinic and quinic derivatives, and 17 were coumarins and coumarin derivatives (Supplementary Table 1).

We first applied principal component analysis (PCA) and orthogonal partial least squares discriminant analysis (OPLS-DA) on these metabolites to discover the differences in metabolites within and between sample groups. The PCA and OPLS-DA results showed that the metabolites of different genotypes and treatments were significantly different and could be used for further analysis (Supplementary Fig. 1A, B). We then performed a normalized clustering analysis and Pearson's correlation analysis to analyze the homogeneity of the biological replicates of each group (Supplementary Fig. 1C, D). The second biological replicate of GL-3 under the control conditions was removed due to a relatively low coefficient $(<0.9)$.

To determine the differentially regulated metabolites, we performed a normalized clustering analysis (Fig. 1). Under the control conditions, 33 metabolites were found to be upregulated in the $M d M Y B 88 / 124$ RNAi plants compared to those in the GL-3 plants. Furthermore, 89 metabolites were found to be downregulated in the $M d M Y B 88 / 124$ RNAi plants compared to those in the GL-3 plants under the control conditions. Under drought conditions, 40 metabolites were found to be upregulated 


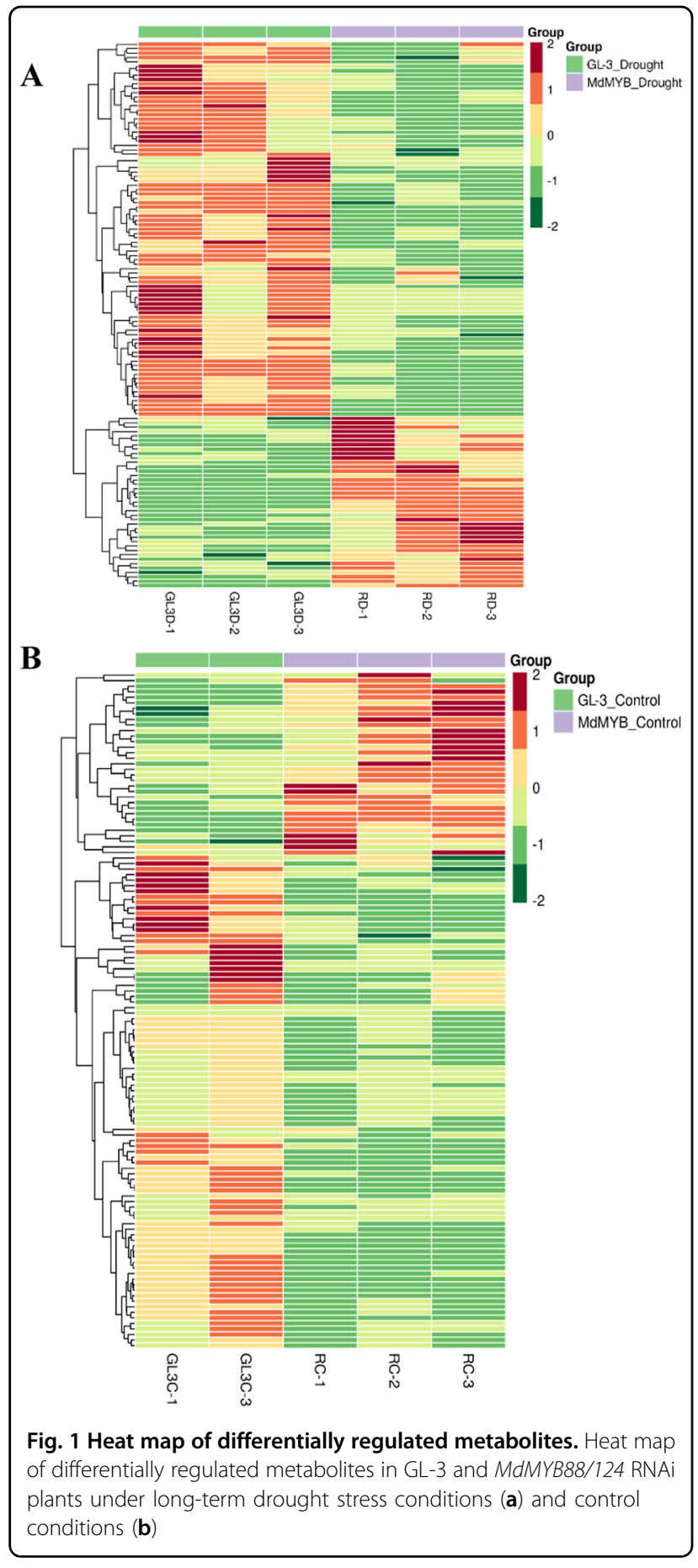

in the MdMYB88/124 RNAi plants compared to GL-3 plants, and 99 metabolites were found to be downregulated. These results suggest that MdMYB88 and MdMYB124 regulate a broad range of secondary metabolites.

KEGG analysis was used to annotate the differentially regulated metabolites in the pathways of phenylpropanoid biosynthesis, flavonoid biosynthesis, and flavone and flavonol biosynthesis (Fig. 2 and Supplementary Figs. 24). All the differential metabolites in these three pathways were found to be downregulated in the MdMYB88/124 RNAi plants under drought conditions compared to those in the GL-3 plants (Fig. 2). These metabolites included 15 involved in the phenylpropanoid biosynthesis pathway, 10 in the flavonoid biosynthesis pathway, and 7 in the flavone and flavonol biosynthesis pathway (Supplementary Figs. $2-4)$. We also discovered that the contents of 10 differential metabolites were downregulated in the MdMYB88/ 124 RNAi plants under the control conditions compared to those in the GL-3 plants (Supplementary Figs. 2-4).

\section{MdMYB88 and MdMYB124 regulate the phenylalanine content in the roots}

The KEGG analysis revealed a large number of differential metabolites related to the phenylpropanoid, flavonoid, flavone and flavonol biosynthesis pathways after drought stress (Fig. 2). Additionally, all these metabolites were downregulated in the roots of $M d M Y B 88 / 124 \mathrm{RNAi}$ lines under drought (Supplementary Table 1); however, under control conditions, a smaller number of differential metabolites were discovered (Supplementary Table 1). These results indicate that MdMYB88 and MdMYB124 play a crucial role in the regulation of the phenylpropanoid, flavonoid, flavone, and flavonol biosynthesis pathways.

Furthermore, the content of phenylalanine in $M d M Y B 88 / 124$ RNAi plants decreased under the control and drought conditions when compared with that in GL-3 plants (Supplementary Table 1). We therefore hypothesized that the downregulation of phenylpropanoids is the result of phenylalanine downregulation. Hence, we verified the content of phenylalanine in the roots of the $M d M Y B 88 / 124$ RNAi lines and GL-3 under both drought and control conditions by the use of UPLC-MS (Fig. 3). Our results showed that the contents of phenylalanine in the roots of the $M d M Y B 88 / 124$ RNAi lines were lower than those in GL-3 under both the control and long-term drought conditions. Alternatively, the phenylalanine content was higher in the roots of the overexpression lines than in GL-3 under both the control and drought conditions (Fig. 3). These findings were consistent with our metabolome results.

The differential content of phenylalanine is interesting. Biosynthesis of phenylalanine in plants occurs through the shikimic acid pathway. An essential reaction in phenylalanine biosynthesis is the conversion of chorismic acid into prephenic acid, catalyzed by $\mathrm{CMs}^{9}$. Three $\mathrm{CMs}$ have been discovered in Arabidopsis ${ }^{42}$, and six homologs in Malus $\mathrm{x}$ domestica were identified: MdCM1, MdCM1like, MdCM2, MdCM2-like, MdCM3, and MdCM3-like (Supplementary Fig. 5). Sequence analysis of the $M d C M$ s revealed a very high similarity between MdCM1 and 


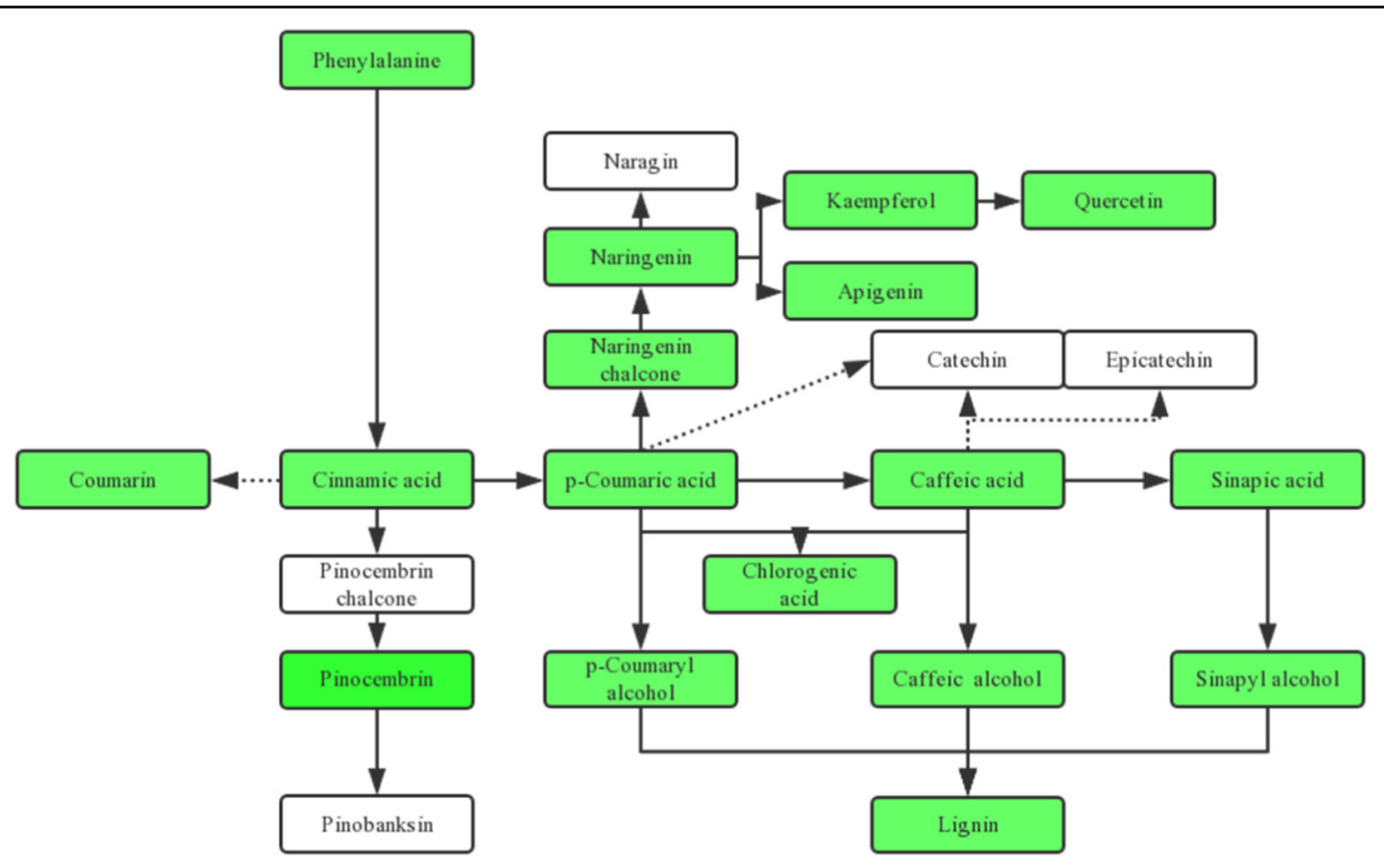

Fig. 2 Differentially regulated metabolites related to the phenylpropanoid and flavonoid biosynthesis pathway under drought. The green color indicates metabolites downregulated in MdMYB88/124 RNAi lines compared with those in GL-3 after long-term drought stress, and the white color represents metabolites that did not change in MdMYB88/124 RNAi lines compared with GL-3 under drought stress

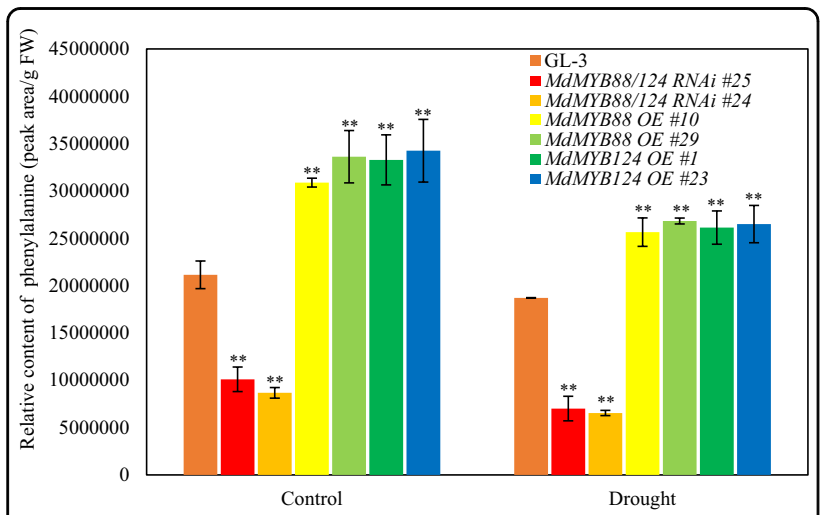

Fig. 3 Content of phenylalanine in the roots of MdMYB88 and MdMYB124 transgenic plants after long-term drought. The data are the means \pm SDs $(n=3)$. One-way ANOVA (Tukey test) was performed, and statistically significant differences are indicated by **(P<0.01). OE overexpression

MdCM1-like, between MdCM2 and MdCM2-like, and between MdCM3 and MdCM3-like. We also noted that MdCM1 is located on chromosome (chr.) 9, and MdCM1-like is located on chr. 16; MdCM2 is located on chr. 5, and MdCM2-like is located on chr. 8; MdCM3 is located on chr. 17, and MdCM3-like is located on chr. 13. The following pairs have homologous segments: chr. 9 and chr. 16, chr. 5 and chr. 8, and chr. 17 and chr. $13^{43}$.
These results indicate that MdCM1 and MdCM1-like, MdCM2 and MdCM2-like, and MdCM3 and MdCM3like are homologs from chromosome doubling.

The expression level of $M d C M$ s in the roots of $M d M Y B 88$ and MdMYB124 transgenic lines treated with $20 \%$ PEG was analyzed by quantitative reverse transcription PCR (qRT-PCR) to determine whether $M d C M$ s were regulated by MdMYB88 and MdMYB124 (Fig. 4). We found that all six homologs were downregulated in $M d M Y B 88 / 124$ RNAi lines and unregulated in $M d M Y B 88$ or MdMYB124 overexpression lines under control and PEG treatment conditions. Promoter analysis revealed the binding site of both MdMYB88 and MdMYB124 (AACCG) in the MdCM2 promoter (Supplementary Fig. 6). Using ChiP-qPCR and Electrophoretic Mobility Shift Assay (EMSA) approaches, we further showed that MdMYB88 can directly bind to the MdCM2 promoter (Fig. 5).

\section{MdMYB88 and MdMYB124 regulate the content of different metabolites in the leaves}

MdMYB88 and MdMYB124 are also expressed in leaves ${ }^{1}$. We therefore examined the accumulation of different metabolites, including flavonoids and phenylpropanoids, in the leaves of the GL-3 and MdMYB88/124 RNAi transgenic plants under both control and drought conditions via a UPLC-MS approach. Among the ten metabolites measured, the contents of chlorogenic acid, 

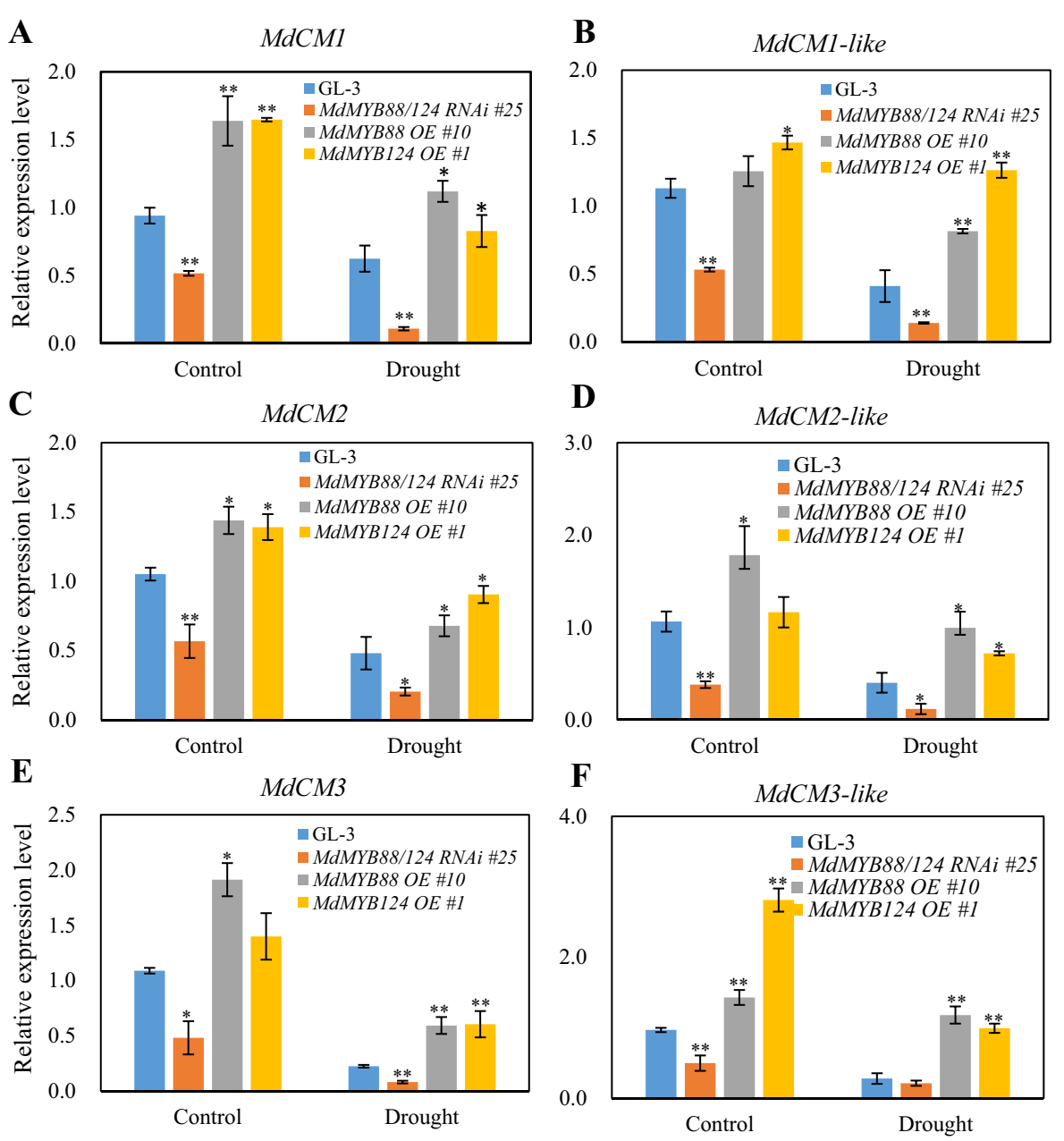

Fig. 4 Relative expression levels of $M d C M s$ in $M d M Y B 88$ and $M d M Y B 124$ transgenic plants under drought stress. Relative expression levels of MdCM1 (a), MdCM1-like (b), MdCM2 (c), MdCM2-like (d), MdCM3 (e), and MdCM3-like (f) in MdMYB88 and MdMYB124 transgenic plants under control and drought conditions. The data are the means \pm SDs $(n=3)$. OE overexpression

catechinic acid, quercetin, and SA (salicylic acid) decreased in the $M d M Y B 88 / 124$ RNAi lines and increased in the $M d M Y B 88$ or $M d M Y B 124$ overexpression lines (Fig. 6). We found no significant differences in the metabolites, including gallic acid, phlorizin, rutin, and caffeic acid (Supplementary Fig. 7).

\section{Different contents of metabolites affect the occurrence of oxidative products and the resistance of pathogens}

Flavonoid and phenylpropanoid metabolites have well-documented relationships with oxidative stress and pathogen resistance ${ }^{44}$. Drought stress is also well known to cause secondary stress, including oxidative stress $^{38}$. We then analyzed the content of hydrogen peroxide $\left(\mathrm{H}_{2} \mathrm{O}_{2}\right)$ and malondialdehyde (MDA) in both the roots and leaves of GL-3, MdMYB88 and $M d M Y B 124$ transgenic plants to examine the occurrence of drought-induced oxidative products. The results showed that there was no difference in the content of $\mathrm{H}_{2} \mathrm{O}_{2}$ or MDA between the GL-3 and transgenic lines under control conditions. There was, however, a significant increase in oxidative products in the $M d M Y B 88 / 124$ RNAi lines and a decrease them in the $M d M Y B 88$ or $M d M Y B 124$ overexpression plants under drought stress (Fig. 7).

Flavonoid and phenylpropanoid metabolites are part of a plant's defense system against pathogenic infection. The different contents of flavonoid and phenylpropanoid metabolites lead to differences in disease resistance ${ }^{45}$. We infected leaves using A. alternata and Valsa mali to evaluate the disease resistance of GL-3, $M d M Y B 88$, and $M d M Y B 124$ transgenic plants in vitro. Our results demonstrate that the leaves of the $M d M Y B 88 / 124$ RNAi lines were sensitive to $A$. alternata, while the leaves of the 


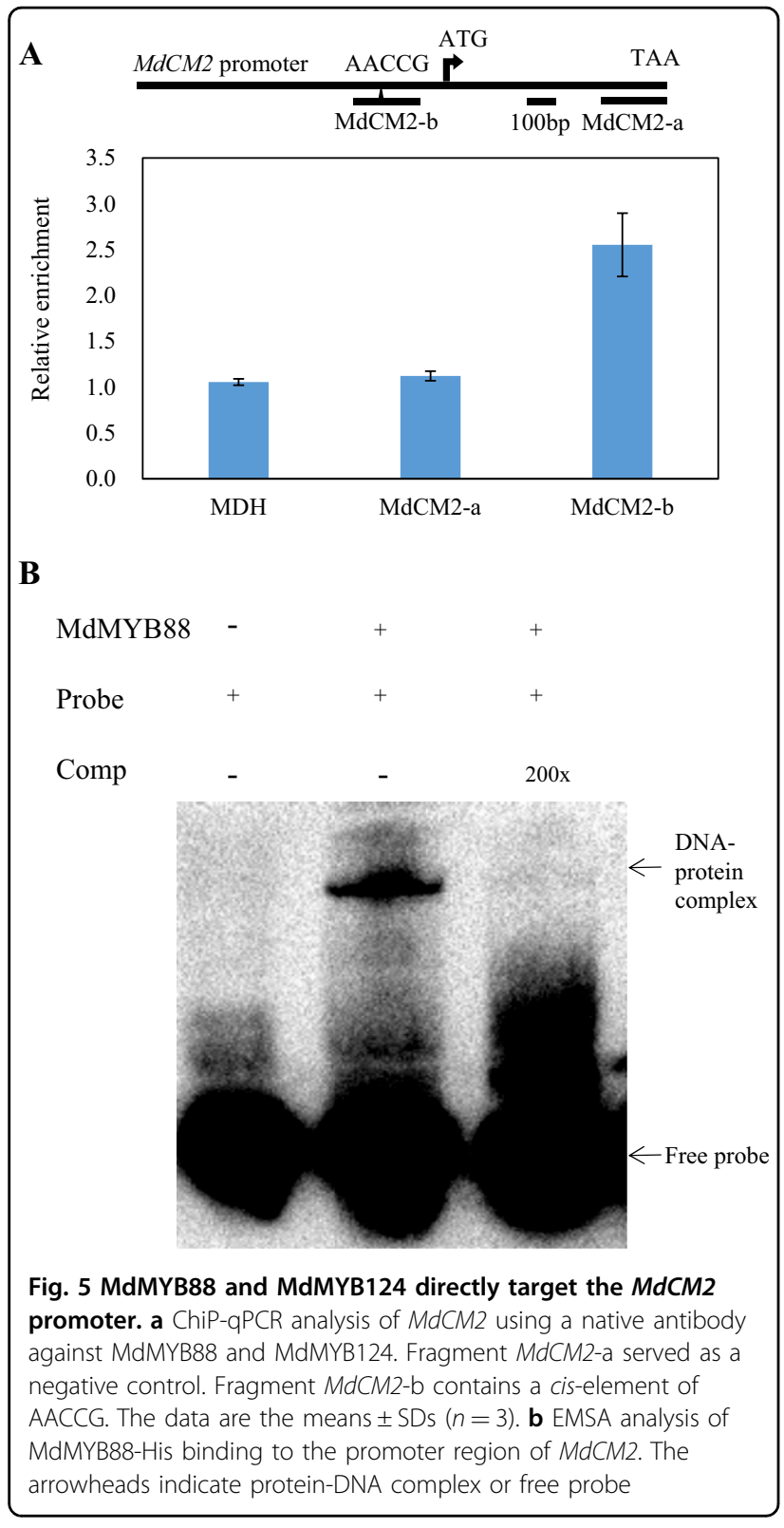

MdMYB88 and MdMYB124 overexpression lines were resistant to $A$. alternata compared with those of GL-3 (Fig. 8). We observed similar patterns in the leaves infected with Valsa mali (Fig. 9).

\section{Discussion}

In previous studies, we presented evidence that MdMYB88 and MdMYB124 regulate both root vessel development and lignin deposition under drought stress in apple trees ${ }^{2}$. We also determined that MdMYB88 and MdMYB124 regulate the expression of genes related to lignin biosynthesis by associating with the MdMYB46 promoter $^{2}$. Lignin is a metabolic compound produced by the phenylpropanoid and flavonoid biosynthesis pathway $^{46}$. In this study, we performed a metabolomic analysis of the roots of GL-3 and $M d M Y B 88 / 124$ RNAi plants under control and drought conditions to understand the metabolic map of MdMYB88 and MdMYB124 and their regulatory roles in the disease resistance of apple trees.

The metabolome analysis revealed that more differentially accumulating metabolites were discovered in the roots of $M d M Y B 88 / 124$ RNAi lines after drought treatment compared to those after the control treatment. A total of 46 different phenylpropanoid and flavonoid metabolites were discovered in the roots of $M d M Y B 88 /$ 124 RNAi plants under the control treatment, while 56 differential phenylpropanoid and flavonoid metabolites were discovered in the $M d M Y B 88 / 124$ RNAi plants under the drought treatment (Supplementary Table 1). The majority of different phenylpropanoid and flavonoid metabolites were downregulated in $M d M Y B 88 / 124$ RNAi plants compared to those in the GL-3 plants (Fig. 2). Furthermore, the content of lignin precursors (including p-coumaryl alcohol, coniferyl alcohol, and sinapyl alcohol) decreased in $M d M Y B 88 / 124$ RNAi plants (Fig. 2). A decrease in the content of all three precursors can lead to a decreased lignin content in $M d M Y B 88 / 124$ RNAi plants, which is consistent with our previous study ${ }^{2}$.

Many flavonoid metabolites, including chlorogenic

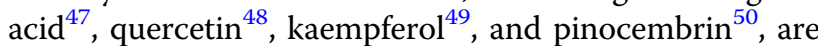
involved in a plant defense responses to fungal and bacterial infections. Derivatives of chlorogenic acid and chlorogenic acid can inhibit the growth of fungi in vivo and in vitro ${ }^{51,52}$. In response to fungal infection, chlorogenic acid in an apple plants can be hydrolyzed into 4hydroxybenzoic acid, which has high antifungal activity, thus increasing the resistance of apple trees to fungi ${ }^{53}$. In immature peach trees and peach trees resistant to Monilinia fructicola, the chlorogenic acid content is exceptionally high ${ }^{54}$. Moreover, in vanilla plants resistant to Fusarium oxysporum, the sinapic acid and hydroxybenzoic acid content is higher than that in sensitive varieties ${ }^{55}$. Here, we discovered that these metabolites were positively regulated by MdMYB88 and MdMYB124, thus indicating that MdMYB88 and MdMYB124 may be involved in fungal resistance. We consistently found that the $M d M Y B 88 / 124$ RNAi plants were more sensitive to $A$. alternata infection than were the other plants, while the $M d M Y B 88$ and MdMYB124 overexpression plants were more tolerant (Figs. 8 and 9).

The phenylpropanoid biosynthetic pathway starts with phenylalanine. CM2 is a critical enzyme involved in phenylalanine biosynthesis ${ }^{42}$. Herein, we determined that the expression of $M d C M 2$ and other $M d C M$ s was positively regulated by MdMYB88 and MdMYB124 (Fig. 4). In addition, MdMYB88 and MdMYB124 could directly bind 
A

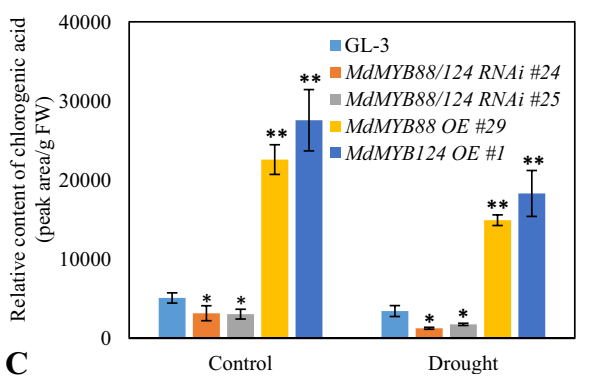

C

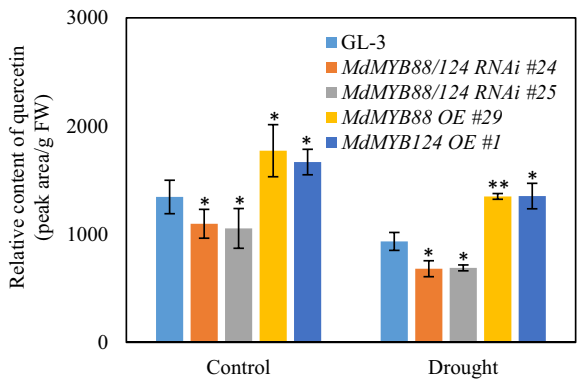

B
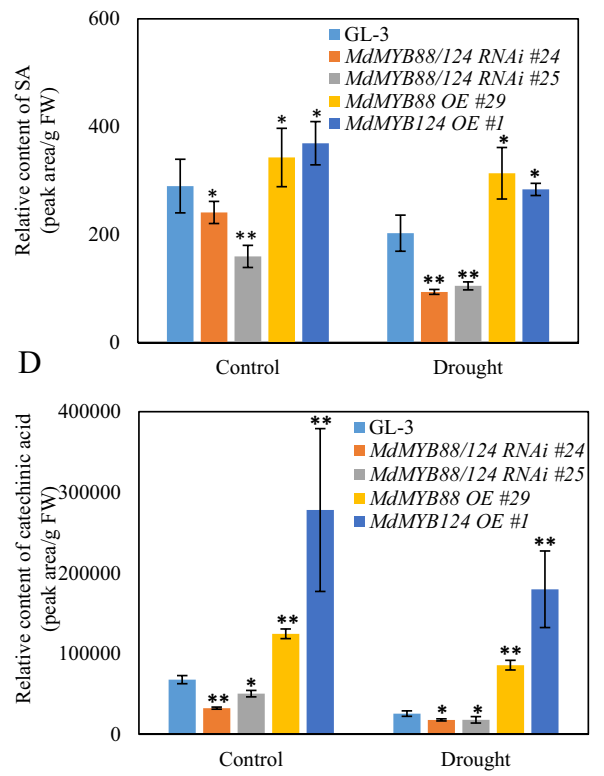

Fig. 6 Content of phenylpropanoids in the leaves of MdMYB88 and MdMYB124 transgentic lines under drought stress. Content of chlorogenic acid (a), SA (b), quercetin (c), and catechinic acid (d) in the leaves under control and long-term drought stress conditions. The data are the means $\pm \operatorname{SDs}(n=3)$. One-way ANOVA (Tukey test) was performed, and statistically significant differences are indicated by $*(P<0.05)$ and $* *(P<0.01)$
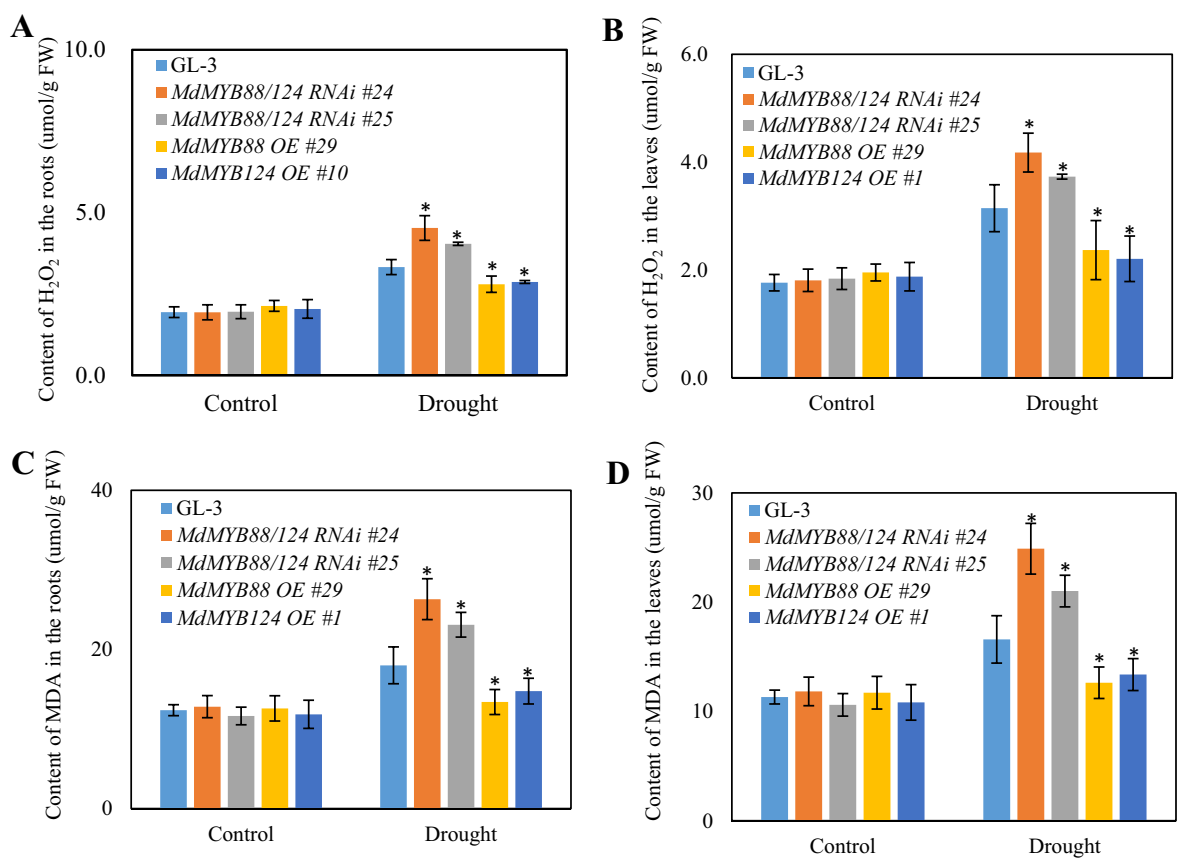

Fig. 7 Content of $\mathrm{H}_{2} \mathrm{O}_{2}$ and MDA in MdMYB88 and MdMYB124 transgentic lines under drought stress. Content of $\mathrm{H}_{2} \mathrm{O}_{2}(\mathbf{a}$ and $\mathbf{b})$ and $M D A$ ( $\mathbf{c}$ and $\mathbf{d}$ ) in the roots (a and $\mathbf{c}$ ) and leaves (b and $\mathbf{d})$ under control and drought stress conditions. The data are the means \pm SDs $(n=5)$. One-way ANOVA (Tukey test) was performed, and statistically significant differences are indicated by $*(P<0.05)$ 
A
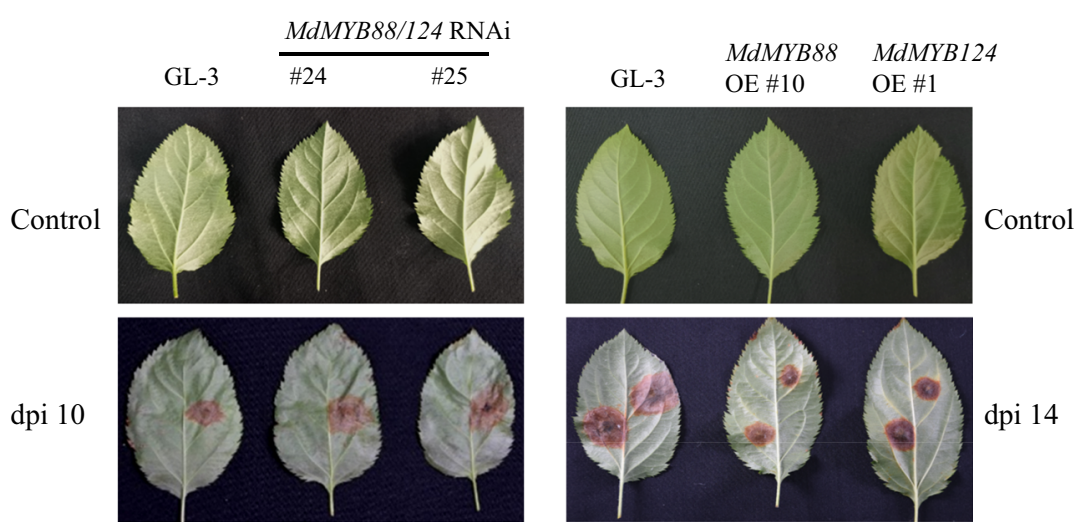

B

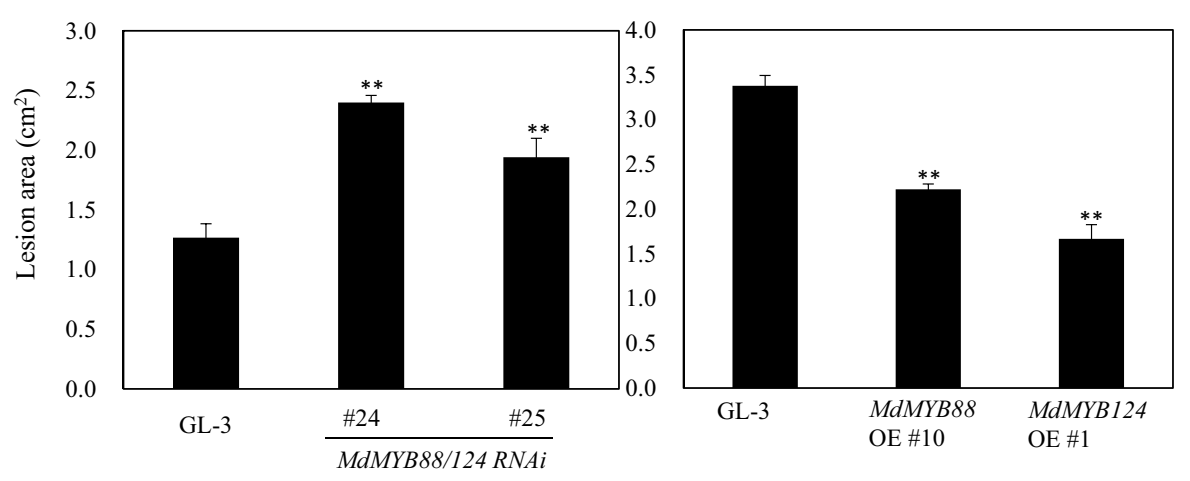

Fig. 8 MdMYB88 and MdMYB124 positively regulate resistance to Alternaria alternata f. sp. mali. a Disease resistance of GL-3, MdMYB88/124 RNAi, MdMYB88, and MdMYB124 overexpression plants (OE). $\mathbf{b}$ Quantification of the data shown in $\mathbf{a}$. The data are the means $\pm S D s(n=8)$. One-way ANOVA (Tukey test) was performed, and statistically significant differences are indicated by ${ }^{* *}(P<0.01)$

to the MdCM2 promoter region (Fig. 5). These results revealed that MdMYB88 and MdMYB124 directly regulate $M d C M 2$. However, we did not find any binding sites of MdMYB88 and MdMYB124 in the promoters of other MdCMs, although MdMYB88 and MdMYB124 positively regulate their expression level. These findings indicate that MdMYB88 and MdMYB124 may regulate other $M d C M$ s through an unknown pathway.

In plants, different phenylpropanoid and flavonoid metabolite contents lead to a difference in the ability of plants to scavenge reactive oxygen species $(\mathrm{ROS})^{56}$. In this study, the $\mathrm{H}_{2} \mathrm{O}_{2}$ and MDA contents were higher in $M d M Y B 88 / 124$ RNAi plants under drought stress than in GL-3 plants; however, there was no significant difference between the GL-3 and the MdMYB88 and $M d M Y B 124$ transgenic lines under the control conditions (Fig. 6). These results indicate that the flavonoid content in the MdMYB88 and MdMYB124 transgenic lines influences the plants' ROS scavenging abilities, which may further contribute to the drought tolerance of apple trees.

In summary, MdMYB88 and MdMYB124 can directly target and induce the expression of $M d C M 2$ and positively regulate other $M d C M$ s. Upregulated $M d C M 2$ and other $M d C M$ s enhance phenylalanine biosynthesis, thus increasing the content of free phenylalanine in both the roots and the leaves. The increased phenylalanine content affects the biosynthesis of downstream metabolites, including phenylpropanoid and flavonoid compounds, which results in increased resistance to oxidative stress and fungal infection.

\section{Methods}

Plant materials, growth conditions, and stress treatment

Tissue-cultured GL-3 [from Malus $\mathrm{x}$ domestica "Royal Gala" seedlings with high regeneration capabilties ${ }^{57}$, transgenic MdMYB88/124 RNAi and MdMYB888 overexpression or MdMYB124 overexpression plants were rooted and transplanted into pots $(30 \mathrm{~cm} \times 18 \mathrm{~cm})$ filled with equal parts of local loess sand and worm cast media. The pots were placed in a greenhouse under natural illumination, with a temperature of $20-35^{\circ} \mathrm{C}$ and a humidity of $35-55 \%$. MdMYB88/124 RNAi plants were generated previously ${ }^{1}$. Owing to the high sequence similarity, both $M d M Y B 88$ and $M d M Y B 124^{1}$ were knocked down in the $M d M Y B 88 / 124$ RNAi plants ${ }^{1}$.

Long-term drought was implemented according to the methods described by Geng et al. ${ }^{2}$. After drought stress, 

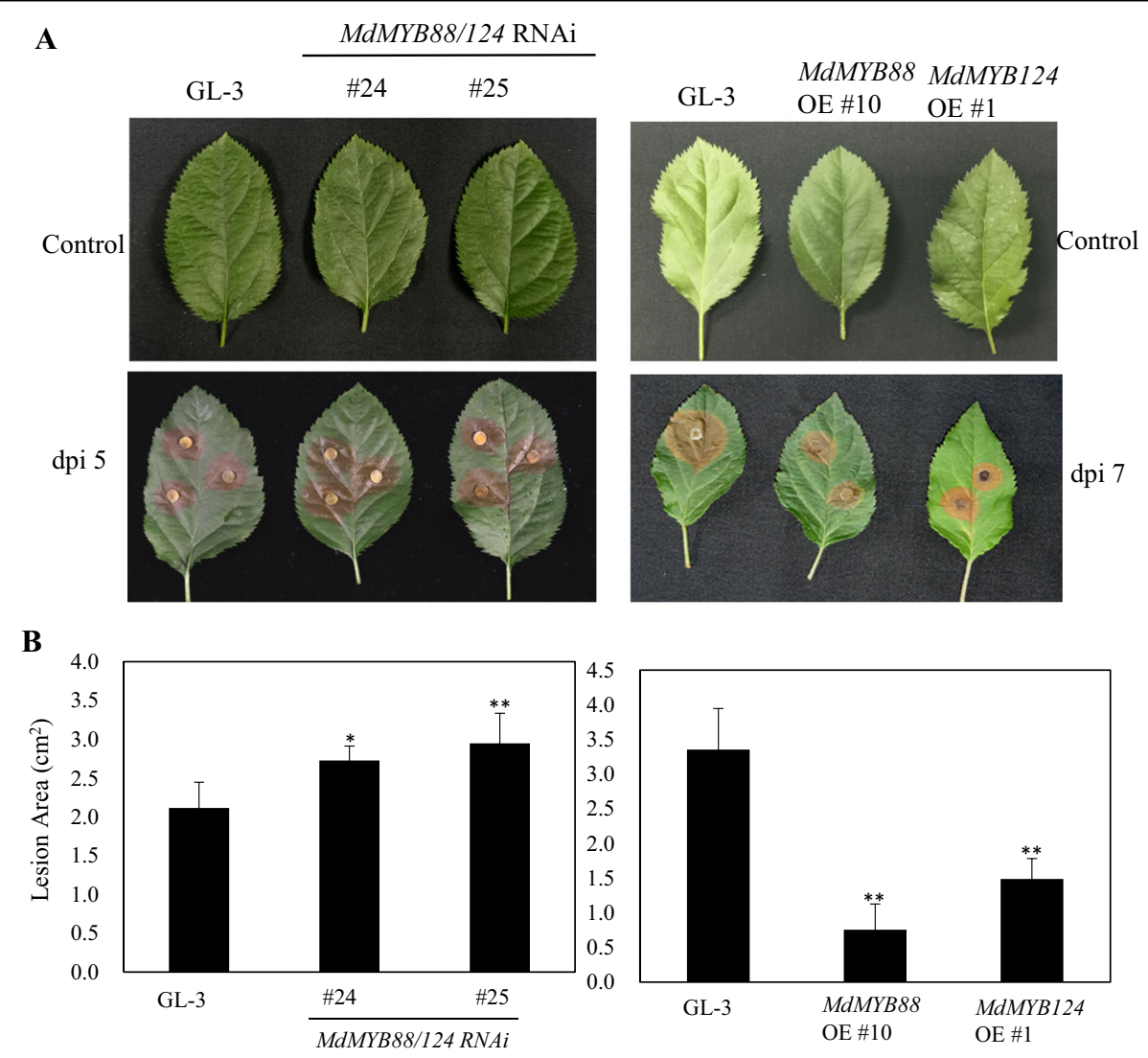

Fig. 9 MdMYB88 and MdMYB124 positively regulate resistance to Vasla mali. a Disease resistance of GL-3, MdMYB88/124 RNAi, MdMYB88, and MdMYB124 overexpression plants (OE). $\mathbf{b}$ Quantification of the data shown in $\mathbf{a}$. The data are the means \pm SDs $(n=8)$. One-way ANOVA (Tukey test) was performed, and statistically significant differences are indicated by $*(P<0.05)$ or $* *(P<0.01)$

the roots were washed and harvested for UPLC-MS analysis.

For disease resistance analysis, GL-3 and MdMYB88 or $M d M Y B 124$ transgenic plants were propagated in MS culture media containing $0.2 \mathrm{mg} / \mathrm{l}$ 6-benzylaminopurine and $0.2 \mathrm{mg} / \mathrm{l}$ indole-3-acetic acid. After 1 month, the transgenic plants were transferred to rooting media containing $0.5 \mathrm{mg} / \mathrm{l} 3$-indolebutyric acid and $0.5 \mathrm{mg} / \mathrm{l}$ indole3 -acetic acid. After 45 days, the plants were transplanted into soil and into a growth chamber with a 16/8-h light/ dark photoperiod. After $\sim 1$ month, the apple plants were ready for the infection experiments.

\section{Disease resistance analysis}

The plant pathogens A. alternata and Valsa mali were isolated previously ${ }^{58}$. The fungi were grown on PDA (potato dextrose agar) media at $25^{\circ} \mathrm{C}$ for $\sim 6$ days. Fifteen to twenty leaves of GL-3 and MdMYB88 or MdMYB124 transgenic plants were collected and washed with $\mathrm{ddH}_{2} \mathrm{O}$ twice, soaked in $6 \%$ sodium hypochlorite solution in the dark for approximately $5 \mathrm{~min}$ and then rewashed with $\mathrm{ddH}_{2} \mathrm{O}$. The leaves were then infected using bacterial plaque obtained by a hole puncher to maintain consistency. After infection, the leaves were returned to the culture dishes and placed in an incubator (70\% humidity; $25-28^{\circ} \mathrm{C}$ ) for two weeks. Afterward, images were taken, and the leaf spots were measured by ImageJ software. The experiments were repeated three times, and one-way ANOVA (Tukey test) was performed by SPSS 19.0.

\section{UPLC-MS analysis}

UPLC-MS analysis was performed according to the methods of Chen et al. ${ }^{59}$, with modifications. Freeze-dried roots were crushed using a mixer mill (MM 400, Retsch, German) with silica sand for $2 \mathrm{~min}$ at $60 \mathrm{~Hz}$. One hundred milligrams of powder was then weighed and extracted overnight at $4{ }^{\circ} \mathrm{C}$ with $1.0 \mathrm{ml}$ of $70 \%$ aqueous methanol. Following centrifugation at $10,000 \times g$ for $10 \mathrm{~min}$, the extracts were absorbed (CNWBOND Carbon-GCB SPE Cartridge, $250 \mathrm{mg}, 3 \mathrm{ml}$; Anpel, Shanghai, China) and filtered by a filter membrane with a pore size of $0.22 \mu \mathrm{m}$. The extracts were then analyzed using an Liquid chromatography electrospray ionization tandem mass spectrometry (LC-ESI-MS/MS) system. The analytical 
conditions were as follows: UPLC column, Waters ACQUITY UPLC HSS T3 C18 $(1.8 \mu \mathrm{m}, 2.1 \mathrm{~mm} \times$ $100 \mathrm{~mm})$; solvent system, water $(0.04 \%$ acetic acid):acetonitrile $(0.04 \%$ acetic acid); gradient program, $95: 5 \mathrm{v} / \mathrm{v}$ at $0 \mathrm{~min}, 5: 95 \mathrm{v} / \mathrm{v}$ at $11.0 \mathrm{~min}, 5: 95 \mathrm{v} / \mathrm{v}$ at $12.0 \mathrm{~min}, 95: 5 \mathrm{v} / \mathrm{v}$ at $12.1 \mathrm{~min}$, and $95: 5 \mathrm{~V} / \mathrm{Vv} / \mathrm{v}$ at $15.0 \mathrm{~min}$; flow rate, $0.40 \mathrm{ml} /$ min; temperature, $40{ }^{\circ} \mathrm{C}$; and injection volume, $2 \mu \mathrm{l}$. The effluent was alternatively connected to an ESI-triple quadrupole-linear ion trap (QTRAP)-MS. LIT and triple quadrupole (QQQ) scans were acquired by a API 6500 QTRAP LC/MS/MS triple quadrupole-linear ion trap mass spectrometer (QTRAP) system. The ESI source operation parameters were the same as those in the study by Chen et al. ${ }^{59}$. PCA and Pearson correlation analysis were performed by SPSS 19.0. OPLS-DA, discovery and KEGG annotations were performed by the $\mathrm{R}$ package.

\section{Measurements of $\mathrm{H}_{2} \mathrm{O}_{2}$ and MDA content}

The contents of $\mathrm{H}_{2} \mathrm{O}_{2}$ and MDA were measured by a hydrogen peroxide assay kit (\#BC3590, Solarbio, Beijing, China) and a malondialdehyde assay kit (\#BC0020, Solarbio, Beijing, China), respectively. Five repeats were measured for each line.

\section{RNA extraction and RT-qPCR analysis}

RNA extraction was carried out as described by Xie et al. ${ }^{1}$. RT-qPCR analysis was performed according to the methods of Guan et al. ${ }^{60}$. The primers used are listed in Supplementary Table 2.

\section{EMSAs and ChIP-qPCR}

EMSAs and ChIP-qPCR were performed as described by Xie et al. ${ }^{1}$. The probes used for the EMSAs are listed in Supplementary Table 2.

\section{Acknowledgements \\ We thank Dr. Zhihong Zhang from Shenyang Agricultural University for providing the tissue-cultured GL-3 plants. We thank Dr. Lili Huang from Northwest A\&F Agricultural University for providing Valsa mali. This work was supported by the National Key Research and Development Program of China (2018YFD1000100) and the National Natural Science Foundation of China (31622049, 31872080, and 31660565).}

\section{Conflict of interest}

The authors declare that they have no conflict of interest.

Supplementary Information accompanies this paper at (https://doi.org/ 10.1038/s41438-020-0324-2).

Received: 6 January 2020 Accepted: 16 April 2020

Published online: 01 July 2020

\section{References}

1. Yinpeng, $X$. et al. An atypical R2R3 MYB transcription factor increases cold hardiness by CBF-dependent and CBF-independent pathways in apple. New Phytol. 218, https://doi.org/10.1111/nph.14952 (2017).
2. Geng, D. et al. MdMYB88 and MdMYB124 enhance drought tolerance by modulating root vessels and cell walls in apple. Plant Physiol. 178, 1296 (2018).

3. Yoon, J., Choi, H. \& An, G. Roles of lignin biosynthesis and regulatory genes in plant development. J. Integr. Plant Biol. 57, 902-912 (2015).

4. Vogt, T. Phenylpropanoid biosynthesis. Mol. Plant 3, 2-20 (2010).

5. Vanholme, R., Demedts, B., Morreel, K., Ralph, J. \& Boerjan, W. Lignin biosynthesis and structure. Plant Physiol. 153, 895-905 (2010).

6. Dixon, R. \& Paiva, N. Stress-induced phenylpropanoid metabolism. Plant Cell $\mathbf{7}$ 1085-1097 (1995).

7. Qian, Y. et al. Completion of the cytosolic post-chorismate phenylalanine biosynthetic pathway in plants. Nat. Commun. 10, 15 (2019).

8. Colquhoun, T. A. et al. A petunia chorismate mutase specialized for the production of floral volatiles. Plant J. 61, 145-155 (2010).

9. Cotton, R. \& Gibson, F. The biosynthesis of phenylalanine and tyrosine; enzymes converting chorismic acid into prephenic acid and their relationships to prephenate dehydratase and prephenate dehydrogenase. Biochim. Biophys. Acta. 100, 76-88 (1965).

10. Cotton, R. G. H. \& Gibson, F. The biosynthesis of phenylalanine and tyrosine in the pea (Pisum sativum): Chorismate mutase. Biochim. Biophys. Acta 156, 187-189 (1968)

11. McCalla, D. \& Neish, A. C. Metabolism of phenylpropanoid compounds in Salvia. I. Biosynthesis of phenylalanine and tyrosine. Can. J. Biochem. Physiol. 37 531-536 (1959).

12. Higuchi, T. \& Brown, S. Studies of lignin biosynthesis using isotopic carbon. XIII. The phenylpropanoid system in lignification. Can. J. Biochem. Physiol. 41, 621-628 (1963).

13. Komives, T. \& Casida, J. Diphenyl ether herbicides: effects of acifluorfen on phenylpropanoid biosynthesis and phenylalanine ammonia-lyase activity in spinach. Pestic. Biochem. Physiol. 18, 191-196 (1982).

14. Diniz, T. C. et al. The role of flavonoids on oxidative stress in epilepsy. Oxid. Med. Cell. Longev. 2015, 171756 (2015).

15. Sadeghnezhad, E., Sharifi, M. \& Zare-Maivan, H. Profiling of acidic (amino and phenolic acids) and phenylpropanoids production in response to methyl jasmonate-induced oxidative stress in Scrophularia striata suspension cells. Planta 244, 75-85 (2016).

16. Hritcu, L. et al. Antidepressant flavonoids and their relationship with oxidative stress. Oxid. Med. Cell. Longev. 2017, https://doi.org/10.1155/2017/5762172 (2017).

17. Nakabayashi, R. et al. Enhancement of oxidative and drought tolerance in Arabidopsis by overaccumulation of antioxidant flavonoids. Plant J. 77 367-379 (2014).

18. Treutter, D. Significance of flavonoids in plant resistance and enhancement of their biosynthesis. Plant Biol. (Stuttg.) 7, 581-591 (2005).

19. Hassan, S. \& Mathesius, U. The role of flavonoids in root-rhizosphere signalling: opportunities and challenges for improving plant-microbe interactions. J. Exp. Bot. 63, 3429-3444 (2012).

20. Gill, U. S. et al. Metabolic flux towards the (iso)flavonoid pathway in lignin modified alfalfa lines induces resistance against Fusarium oxysporum f. sp. medicaginis. Plant Cell Environ. 41, 1997-2007 (2018).

21. Li, Y., Wei, W., Zhang, J., Li, G. \& Gao, K. Structures and antipathogenic fungi activities of flavonoids from pathogen-infected Astragalus adsurgens. Nat. Prod. Res. 33, 822-826 (2019).

22. Berdeja, M. et al. Water limitation and rootstock genotype interact to alter grape berry metabolism through transcriptome reprogramming. Hortic. Res. $\mathbf{2}$, 15012 (2015).

23. Cotrozzi, L. et al. Phenylpropanoids are key players in the antioxidant defense to ozone of European ash, Fraxinus excelsior. Environ. Sci. Pollut. Res. Int. 25, 8137-8147 (2018)

24. Tattini, M. et al. Isoprenoids and phenylpropanoids are part of the antioxidant defense orchestrated daily by drought-stressed Platanus $x$ acerifolia plants during Mediterranean summers. N. Phytol. 207, 613-626 (2015).

25. Wei, Y., Zhou, D., Peng, J., Pan, L. \& Tu, K. Hot air treatment induces disease resistance through activating the phenylpropanoid metabolism in cherry tomato fruit. J. Agric. Food Chem. 65, 8003-8010 (2017).

26. Yin, Z. et al. Validation of reference genes for gene expression analysis in Valsa mali var. mali using real-time quantitative PCR. J. Microb. Biotechnol. 29, https:/ doi.org/10.1007/s11274-013-1320-6 (2013).

27. Parmar, N. et al. Genetic engineering strategies for biotic and abiotic stress tolerance and quality enhancement in horticultural crops: a comprehensive review. 3 Biotech 7, 239 (2017). 
28. Kwiecien, S. et al. Lipid peroxidation, reactive oxygen species and antioxidative factors in the pathogenesis of gastric mucosal lesions and mechanism of protection against oxidative stress-induced gastric injury. J. Physiol. Pharmacol. 65, 613-622 (2014).

29. Zhang, C. X., Tian, Y. \& Cong, P. H. Proteome analysis of pathogen-responsive proteins from apple leaves induced by the Alternaria Blotch Alternaria alternata. PLOS ONE 10, e0122233 (2015).

30. Cao, K. et al. Investigations on the occurrence and control of apple canker in China. Plant Prot. 35, 114-117 (2009).

31. Abe, K., Kotoda, N., Kato, H. \& Soejima, J. Genetic studies on resistance to Valsa canker in apple: genetic variance and breeding values estimated from intraand inter-specific hybrid progeny populations. Tree Genet. Genomes 7, 363-372 (2011).

32. Vasilyeva, L. \& Kim, W. G. Valsa mali Miyabe et Yamada, the causal fungus of apple tree canker in east Asia. Mycobiology 28, 153-157 (2000).

33. Meng, X. L. et al. Latent infection of Valsa Mali in the seeds, seedlings and twigs of crabapple and apple trees is a potential inoculum source of Valsa Canker. Sci. Rep. 9, 7738 (2019).

34. Ren, Y. R. et al. MdGRF11, an apple 14-3-3 protein, acts as a positive regulator of drought and salt tolerance. Plant Sci. 288, 110219 (2019).

35. Jia, D. et al. An apple (Malus domestica) NAC transcription factor enhances drought tolerance in transgenic apple plants. Plant Physiol. Biochem. 139, 504-512 (2019).

36. Chen, Q. et al. Overexpression of an apple LysM-containing protein gene, MdCERK1-2, confers improved resistance to the pathogenic fungus, Alternaria alternata, in Nicotiana benthamiana. BMC Plant Biol. 20, 146 https://doi.org/ 10.1186/s12870-020-02361-Z

37. Zhang, Y. L. et al. Apple AP2/EREBP transcription factor MdSHINE2 confers drought resistance by regulating wax biosynthesis. Planta 249, 1627-1643 (2019).

38. Mahajan, S. \& Tuteja, N. Cold, salinity and drought stresses: an overview. Arch. Biochem. Biophys. 444, 139-158 (2005).

39. Ding, $Y$. et al. Four distinct types of dehydration stress memory genes in Arabidopsis thaliana. BMC Plant Biol. 13, 229 (2013).

40. Xie, Z., Li, D., Wang, L., Sack, F. \& Grotewold, E. Role of the stomatal development regulators FLP/MYB88 in abiotic stress responses. Plant J.: Cell Mol. Biol. 64, 731-739 (2010).

41. Yanhui, $C$. et al. TheMYB transcription factor superfamily of Arabidopsis: expression analysis and phylogenetic comparison with the rice MYB family. Plant Mol. Biol. 60, 107-124 (2006).

42. Eberhard, J. et al. Cytosolic and plastidic chorismate mutase isozymes from Arabidopsis thaliana: molecular characterization and enzymatic properties. Plant J. 10, 815-821 (1996).

43. Velasco, R. et al. The genome of the domesticated apple (Malus $\times$ domestica Borkh.). Nat. Genet. 42, 833-839 (2010).

44. Duthie, G. \& Crozier, A. Plant-derived phenolic antioxidants. Curr. Opin. Clin. Nutr. Metab. Care 3, 447-451 (2000).
45. Zaynab, M. et al. Role of secondary metabolites in plant defense against pathogens. Microb. Pathog. 124, https://doi.org/10.1016/j.micpath.2018.08.034 (2018).

46. Boerjan, W., Ralph, J. \& Baucher, M. Lignin biosynthesis. Annu. Rev. Plant Biol. 54 519-546 (2003)

47. Martinez, G. et al. Chlorogenic acid is a fungicide active against phytopathogenic fungi. Pestic. Biochem. Physiol. 140, 30-35 (2017).

48. Takahama, U. \& Hirota, S. Deglucosidation of quercetin glucosides to the aglycone and formation of antifungal agents by peroxidase-dependent oxidation of quercetin on browning of onion scales. Plant Cell Physiol. 41, 1021-1029 (2000).

49. Kolisetty, S. R., Babu, G. \& Ramnareddy, Y. Acylated flavone glycosides from the roots of Saussurea lappa and their antifungal activity. Molecules 12, 328-344 (2007).

50. Shain, L. \& Miller, J. B. Pinocembrin: an antifungal compound secreted by leaf glands of eastern cottonwood. Phytopathology 72, 877-880 (1982).

51. Ma, C. M., Kully, M., Khan, J. K., Hattori, M. \& Daneshtalab, M. Synthesis of chlorogenic acid derivatives with promising antifungal activity. Bioorg. Med. Chem. 15, 6830-6833 (2007)

52. Sung, W. \& Lee, D. Antifungal action of chlorogenic acid against pathogenic fungi, mediated by membrane disruption. Pure Appl. Chem. 82, 219-226 (2010).

53. Fawcett, C. \& Spencer, D. Sclerotinia fructigena infection and chlorogenic acid content in relation to antifungal compounds in apple fruits. Ann. Appl. Biol. 61, 245-253 (1968).

54. Lee, M. - H. \& Bostock, R. Fruit exocarp phenols in relation to quiescence and development of Monilinia fructicola infections in Prunus spp:: a role for cellular redox? Phytopathology 97, 269-277 (2007).

55. Koyyappurath, S. et al. Differential responses of vanilla accessions to root rot and colonization by Fusarium oxysporum f. sp. radicis-vanillae. Front. Plant Sci. 6, 1125 (2015).

56. Agati, G., Azzarello, E., Pollastri, S. \& Tattini, M. Flavonoids as antioxidants in plants: location and functional significance. Plant Sci. 196, 67-76 (2012).

57. Dai, H. et al. Development of a seedling clone with high regeneration capacity and susceptibility to Agrobacterium in apple. Sci. Hort. 164, 202-208 (2013).

58. Zhang, Q. et al. A single-nucleotide polymorphism in the promoter of a hairpin RNA contributes to Alternaria alternata leaf spot resistance in apple (Malus x domestica). Plant Cell 30, 1924-1942 (2018).

59. Chen, W. et al. A novel integrated method for large-scale detection, identification, and quantification of widely targeted metabolites: application in the study of rice metabolomics. Mol. Plant 6, 1769-1780 (2013).

60. Guan, Q., wu, J., Yue, X., Zhang, Y. \& Zhu, J. A nuclear calcium-sensing pathway is critical for gene regulation and salt stress tolerance in Arabidopsis. PLOS Genet. 9, e1003755 (2013). 